\title{
Pragmatic Constraints on (Adverbial) (Temporal) Quantification'
}

\author{
Orin Percus \\ University of Milan \\ Orin.Percus@unimi.it
}

\begin{abstract}
Even if we can generate a logical form, principles of use may limit the ways in which we can use it. In this paper, I motivate one such principle of use, and explore its effects. Much of the discussion involves kinds of sentences that have received attention in the literature on "individual-level predicates."
\end{abstract}

\section{A possible line of argumentation, and reasons to reject it}

Here is a familiar line of argumentation similar to one advanced by Kratzer 1995. The argument starts from the idea that in the logical form of a sentence containing an adverbial quantifier like always, the quantifier bears an index. The logical form for a sentence like (1a) on this approach is something like (1b). (And on this approach logical forms that conform to the schema $\left[\alpha \quad\left[\ldots\right.\right.$ always $\left.\left._{1 . .}\right] \quad \beta\right]$ are interpreted roughly as in (1c).) The argument calls attention to the fact that, unlike sentences like those in (1a), sentences like those in (2) sound bizarre. It points out that we can explain why they sound bizarre if we assume, along with some other assumptions, that their possible logical forms contain no item that can be coindexed with the quantifier. It concludes on this basis that indeed (2) contains no item that can be coindexed with the quantifier.

(1) a. Ingrid is always on the phone.

b. If: $[\alpha[\ldots$ always $1 . .].[\beta \ldots 1 \ldots$ Ingrid on the phone $\ldots]$

c. $[[\alpha]]^{\mathrm{g}}(\mathrm{w})=1$ as long as for all time intervals $t$ that satisfy in $w$ the contextually salient property of time intervals, $[[\beta]]^{\mathrm{g} 1->\mathrm{t}}(\mathrm{w})=1$.

(2) Ingrid was always Swedish.

My hope in this paper is to defeat this line of argumentation. My thesis is that there are principles of grammar that (2) violates even if we analyze it as containing an item that can be coindexed with the quantifier. If this is correct, then just on the basis of the strangeness of (2), we have no evidence for the claim that its logical form lacks these additional indices (or for that matter for the assumptions that derive the bizarreness of (2) given the lack of additional indices). Maybe there is evidence for something like this, but it doesn't come from sentences like (2).

My main goal in this paper is to independently motivate a principle from which it happens to follow that (2) sounds bizarre. I will demonstrate some of the consequences of this principle, and I will conclude by suggesting that once we recognize this principle, we gain some insight into the question of what interpretations sound natural for sentences with indefinites. As far as this workshop is concerned, this paper should serve as a cautionary note.

\footnotetext{
${ }^{1}$ This is the first draft ever of the material under discussion, and likely contains many errors. Comments and corrections are more than welcome. Thanks to the ZAS Workshop on Predicative Constructions for the opportunity to present the work here, and to Andrea Bonomi, Gennaro Chierchia and Manfred Krifka for related chats.
} 
To the extent that the pragmatic principle here can answer the question of why sentences like (2) sound bizarre and what interpretations sound natural for sentences with indefinites, we should not use the same facts to motivate syntactic stipulations that do the same work.

To see why I think that (2) incurs problems that should not be traced to the presence or absence of an item coindexed with the quantifier, consider the sentence in (3).

The student who finished first was always Swedish.

Imagine that we were both present at a series of exams, which took place from Monday through Saturday. We both saw that each time a different person finished first. In fact, the pattern was as in (4a):

We both see:

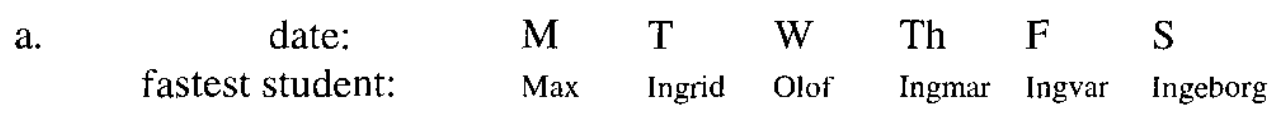

You just saw the students, you don't know who they were or what their nationalities were, and so I tell you (3) to inform you of their nationalities. There is nothing bizarre about (3) when used in this context. What can we conclude from this? If the absence of an item coindexed with the quantifier would render the sentence bad, and in fact the sentence is good, we can conclude that the sentence admits a logical form containing an item coindexed with the quantifier.

Now that we have established that (3) admits a logical form containing an item coindexed with the quantifier, imagine a different situation. Just as before, we were both present at the exams, but this time the pattern is different:

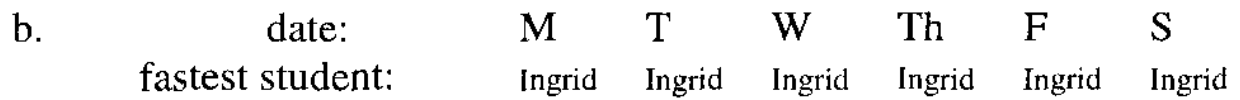

Since we were both there, we both saw that the same person finished first each time. Still, you don't know who it was or what her nationality was. In this context, I cannot use (3) to inform you of the student's nationality. It sounds bizarre. And, since we have already concluded that the sentence admits a logical form containing an item coindexed with the quantifier, it can't be that its bizarreness is due to the lack of such a logical form.

I think that it is reasonable to expect that the same thing that renders (3) bizarre in this context renders (2) bizarre. That is why I think that (2) incurs problems that have nothing to do with the lack of an item that can be coindexed with the quantifier.

A similar point can be made by considering sentences like (5). Unlike the sentence in (6), which sounds fine and therefore must contain an item coindexed with the quantifier, (5) sounds bizarre, at least on an initial reading and encountered in isolation.

John always knows whether Ingrid is Swedish.

(6) John always knows whether Ingrid is on the phone.

Now, when it comes to the sentence in (6), it can be argued not only that the quantifier is coindexed with another item, but more specifically that it is coindexed with an item in the 
matrix clause. (I will sketch how in a moment.) On the assumption that the only syntactic difference between (5) and (6) is the embedded clause, this means that (5) too must contain an item in the matrix clause that always can be coindexed with. So it can't be that the bizarreness of (5) is due to the lack of this kind of item.

I think that it is reasonable to expect that the same thing that renders (5) bizarre renders (2) bizarre, and so that is another reason for thinking that (2) incurs problems that have nothing to do with the lack of an item that can be coindexed with the quantifier. ${ }^{2}$

The argument that sentences like (6) contain an indexed item in the matrix is complicated but its rough outline is as follows. It assumes that the possible logical forms for (6) are as in (7b)
a. John always knows whether Ingrid is on the phone.
b. $\left[\ldots\right.$ always $\left.{ }_{i} \ldots\right][\beta \ldots$ John know whether... $[\gamma \quad]$ ]

where the embedded constituent $\gamma$ is the same kind of constituent that you get in the logical form of simpler sentences like Ingrid is always on the phone, a constituent that contains an index:
a. Ingrid is always on the phone.
b. $\left[\ldots\right.$...always $\left.\mathrm{a}_{\mathrm{i}} \ldots\right][\gamma \ldots \mathrm{i}$...Ingrid on the phone $]$

On the basis of this, it argues that to make the right predictions about the semantics of sentences like (7a) and ( $8 \mathrm{a}$ ), the constituents $\beta$ and $\gamma$ must be interpreted as follows:

$$
\begin{gathered}
{[[\beta]]^{g}=\lambda w . \text { if, in } w, \text { Ingrid is on the phone for the duration of } g(i)} \\
\text { then, in } w, \text { John for the duration of } g(i) \text { believes Ingrid } \\
\text { to be on the phone; and } \\
\text { if, in } w \text {, Ingrid is not on the phone for the duration of g(i) } \\
\text { then, in } w \text {, John for the duration of } g(i) \text { believes Ingrid } \\
\text { not to be on the phone }
\end{gathered}
$$

(abbreviated further: $\lambda w$. in $w$, for the duration of $g(i)$ John knows whether Ingrid is on the phone)

(10) $[[\gamma]]^{g}=\lambda w$. in $w$, Ingrid is on the phone for the duration of $g(i)$

It is important to note here that, in the semantics of $\beta, \mathrm{g}(\mathrm{i})$ plays a role in determining the duration of John's beliefs.

The argument then points out that, on certain ideas about semantic composition, to say that the matrix clause in ( $7 \mathrm{~b})$ does not contain an indexed item amounts to saying that the matrix clause material behaves semantically like a function that, given the denotation that you get for $\gamma$, will yield the denotation that you get for $\beta$. That is, it will behave like a function that, for any arbitrary assignment $g$ and index i such that $g(i)$ yields a time interval, will take a proposition of the kind in (10) and give you a proposition of the kind in (9).

\footnotetext{
${ }^{2}$ See de Swart 1991 for arguments of a similar nature.
} 
(11) The matrix clause material "behaves like" some function F with the characteristic that,

for any time interval $y$,

$\mathrm{F}$ ( $\lambda w$. in $w$, Ingrid is on the phone for the duration of $y$ )

$=\lambda \mathrm{w}$. in $\mathrm{w}$, for the duration of $\mathrm{y}$ John knows whether Ingrid is on the phone.

But at this point problems arise. On the one hand, one can make a case that it is implausible to assume that the matrix clause behaves like this. On the other hand, assuming that the matrix clause behaves like this seems to make wrong predictions. Therefore, we do not want to say that the matrix clause in (7b) lacks an indexed item.

The wrong predictions that the proposal in (11) makes are as follows. Parallel to the pair of sentences in (7) and (8), we have pairs of sentences like those in (12a) and (13a), for which we would posit parallel logical forms:

(12) a. John always knows whether at $5 \mathrm{pm}$ on the following day Ingrid is on the phone.

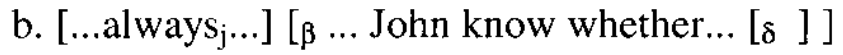

(13) a. At 5pm on the following day Ingrid is always on the phone.

b. [...always $\left.\mathrm{j}_{\mathrm{j} . . .}\right][\delta \ldots \mathrm{j} . .$. at $5 \mathrm{pm}$ on the following day Ingrid on the phone ]

Given the way (13a) is interpreted, the right semantics for $\delta$ seems to be:

$[[\delta]]^{\mathrm{g}}=\lambda \mathrm{w}$. in $\mathrm{w}$, Ingrid is on the phone at $5 \mathrm{pm}$ on the day after $\mathrm{g}(\mathrm{j})$

Now by assumption to obtain $[[\beta]]^{\mathrm{g}}$ we apply the function $\mathrm{F}$ to $[[\delta]]^{\mathrm{g}}$. So take an arbitrary assignment $\mathrm{g}$ and index $\mathrm{j}$. What will $\mathrm{F}$ yield for the proposition in (14)? $\mathrm{F}$ will yield:

$$
\begin{aligned}
& {[[\beta]]^{g}=F\left([[\delta]]^{g}\right)=} \\
& \lambda w \text {. in } w, \text { at } 5 \mathrm{pm} \text { on the day following g(j), John knows whether Ingrid is } \\
& \text { on the phone }
\end{aligned}
$$

Glossing over some steps, the consequence will be that a sentence like (12a) should express that all relevant times are such that John knows one day later at $5 \mathrm{pm}$ whether Ingrid is on the phone. But it doesn't express that. It expresses that all relevant times are such that John knows then whether one day later at $5 \mathrm{pm}$ Ingrid is on the phone.

\section{What is the generalization about when $(3)(=(16))$ can be used?}

I will now return to the sentence in (16), and attempt to describe the conditions under which it is infelicitous. On the basis of this description, I will then propose a principle of pragmatics that determines when it is appropriate to use sentences of this kind.

The student who finished first was always Swedish.

The discussion in this section will rely on some assumptions that I will make about the logical forms available for sentences like (16) and about aspects of the way these logical forms are interpreted. These assumptions are intentionally simplified, and in the rest of the paper I will take the simplifications for granted. I will hope that on different assumptions, the essence of 
what I have to say will remain even though the details will have to be different. The assumptions are these:

First, as far as the syntax of sentences like (16), I will be consistent with what I have been assuming until now. I will assume that in the lfs of these sentences all items have reconstructed to below the VP level, and I will be assuming that there is nothing interpretable above always, which is adjoined to VP, so that everything above always can be ignored. (This is a big simplification for one thing because it means that I am ignoring tense nodes, and thus tense information.) As before, I will assume that adverbial quantifiers like always are indexed. The interpretable pieces of (16)'s If are thus as in (17).

$\operatorname{always}_{1}[\alpha]$

When it comes to my assumptions about how lfs like (17) are interpreted, I will be departing slightly from an idea that I alluded to earlier (in (1c)). Earlier, I assumed that the context makes salient a property of time intervals, and that we use this property to determine the intervals that always quantifies over (informally speaking). Now, I will assume that the context makes salient a set of time intervals, and that we interpret quantifiers like always as quantifying over the members of this set. Specifically, an lf like (17) will be interpreted as in (18). This simplification will strongly affect the terms of the coming discussion.

$\left[\left[\operatorname{always}_{\mathrm{i}}[\alpha]\right]\right]^{\mathrm{g}}=$

$\lambda \mathrm{w}$. for all time intervals $t$ in the contextually salient set $S$ of time intervals, $[[\alpha]]^{\mathrm{gi}->\mathrm{t}}(\mathrm{w})=1$.

In general, interpretation will work in such a way that, when we compute the denotation of a sentence's If with respect to an assignment, we will get a function from worlds to truth values. The way in which these denotations fit into a theory of the way truth judgments depend on syntactic structures is the usual one: on this theory, when we say that a sentence is true, we are saying that we can find an If for it and an assignment such that the actual world is characterized by the function we get by evaluating the If with respect to the assignment.

Once we make assumptions like these, we can draw conclusions about other aspects of the semantics of (16). Consider once again sentence (16) as uttered in the first scenario, on which the student who finishes first is different each time.

$$
\begin{array}{lllllll}
\text { Exam date: } & \mathrm{M} & \mathrm{T} & \mathrm{W} & \mathrm{Th} & \mathrm{F} & \mathrm{S}
\end{array}
$$

Fastest student: a $\quad$ b $\quad$ c $\quad$ d $\quad$ e $\quad f$

The fact is that, if we know that the students who finished were all Swedish-born, we would say that the sentence is true. If we assume that the If of (16) conforms to the format in (17), then we might draw the following conclusions from this. First, the context makes salient a series of exam days $((20 a))$ - specific time intervals during which the actual exams occurred. Second, in the If of (16), the denotation of the constituent that combines with always is as in (20b).

a. $S=\{$ Monday, Tuesday, Wednesday, Thursday, Friday, Saturday $\}$ 
b. $[[\alpha]]^{g}=\lambda w$. the individual who in $w$ is the student first to finish the exam held in $g(1)$ is Swedish-born in w.

With this, we account for the fact that, if we think that the students who finished first were all Swedish, then we take the sentence to be true. (Reasoning: Take any assignment. Given (18) and (20), the function that we get by evaluating the If of (16) with respect to that assignment will be as in (21) below. This function characterizes the actual world as long as on each exam day the first student to finish was Swedish. Now, by hypothesis, to say that (16) is true is to say that the actual world is characterized by a function that we get when we evaluate (16)'s If with respect to some assignment. So suppose that we think that the actual world is such that on each exam day the first student to finish was Swedish. Well, this amounts to saying that we think that the actual world is characterized by the function in (21). So given that the function in (21) is a function that we get by evaluating (16)'s If with respect to some assignment, we should take (16) to be true.)

(21) $\lambda w$. For all days $t$ in $S$, the individual who in $w$ is the student first to finish the exam held in $t$ is Swedish-born in $w$.

$\left(=[[(17)]]^{g}\right.$ for all g)

In what follows, I will assume that these are the right conclusions to draw. If these are the right conclusions to draw, then to say that (16) is true is to say that the function in (21) characterizes the actual world. Equivalently, to say that (16) is true is to say that all of the functions in $\Sigma$ characterize the actual world:

(22) $\lambda w$. the individual who in $w$ is the student first to finish the exam held on MONDAY is Swedish-born in w. I $\lambda w$. the individual who in $w$ is the student first to finish the exam held on TUESDAY is Swedish-born in $w$. |

$\Sigma=\{\lambda w$. the individual who in $w$ is the student first to finish the exam held on WEDNESDAY is Swedish-born in $w$. $\}$

| $\lambda w$. the individual who in $w$ is the student first to finish the exam held on THURSDAY is Swedish-born in $w$. |

$\lambda \quad \lambda w$. the individual who in $w$ is the student first to finish the exam held on FRIDAY is Swedish-born in $w$.

( $\lambda w$. the individual who in $w$ is the student first to finish the exam held on SATURDAY is Swedish-born in $w$. J

Now, here is the generalization that I am proposing specifically about when (16) can be used. This generalization makes reference to the function in (21). On the assumptions so far, (21) is the denotation of (16)'s if with respect to any assignment, so on the assumptions so far the generalization makes reference to the denotation of (16)'s If.

Proposed generalization about (16):

The cases where (16) sounds strange are the cases where we could determine whether (21) holds of the actual world by determining whether the propositions in some proper subset of $\Sigma$ hold of the actual world. (And moreover - as will be discussed below -the smaller the subset, the worse (16) sounds.)

Consider for example our second scenario, repeated in (24).

Exam date:

Fastest student:

$$
\text { M }
$$

a

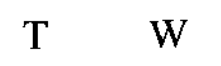

a

a

Th

a

F

\section{$S$}

a a

We were both present at all the exams, and I tell you (16) afterwards to inform you of a's nationality. 
At the point when I utter this sentence, we are both aware that the student who finished first is the same for all $t$ in $S$. That is,

(25) We are both aware that the actual world w0 has the following property:

For all $\mathrm{t} 1, \mathrm{t} 2$ in $\mathrm{S}$, the individual who in w0 is the student first to finish the exam in $\mathrm{t} 1$ is the individual who in $w 0$ is the student first to finish the exam in $t 2$.

This means that, to determine whether (21) holds of the actual world, it is enough to determine whether just one of the propositions in $\Sigma$, say (26), holds of the actual world.

$\lambda w$. the individual who in $w$ is the student first to finish the exam held on TUESDAY is Swedish-born in w.

(Suppose it does. Then

(27) the individual who in w0 is the student first to finish the exam held on Tuesday is Swedish-born in w0.

And then from (25) - omitting some steps -- it will follow that

(28) For all days $t$ in $S$, the individual who in w0 is the student first to finish the exam held in $\mathrm{t}$ is Swedish-born in w0.

i.e. that (21) holds of the actual world. On the other hand, suppose it doesn't. Then

(27') the individual who in w0 is the student first to finish the exam held on Tuesday is NOT Swedish-born in w0.

And then from (25) it will follow that

(28') For all days $t$ in $\mathrm{S}$, the individual who in w0 is the student first to finish the exam held in $t$ is NOT Swedish-born in w0.

i.e. that (21) does not hold of the actual world.)

Here, then, we can determine whether (21) holds of the actual world by considering just a singleton subset of $\Sigma$. And the intuition is that (16) is very strange.

I think that, when we consider variations on the scenario that we have been considering, we find that the strangeness of (16) is (inversely) related to the size of the smallest subset of $\Sigma$ that can serve to verify (21). Imagine, for example, the variation in (29i), on which we see one student finishing first every day from Monday through Wednesday, and a different student finishing first every day from Thursday through Saturday. The intuition, I think, is that in this slightly altered scenario it is still quite strange to utter (16), but perhaps not quite as strange as in the scenario on which the same student finishes first each time. In this new case, we can't determine whether (21) holds of the actual world by considering a singleton subset of $\Sigma$, but we can by considering a two-membered subset - for example, $\{\lambda w$. the individual who in $w$ is the student first to finish the exam held on TUESDAY is Swedishborn in $w, \lambda w$. the individual who in $w$ is the student first to finish the exam held on FRIDAY 
is Swedish-born in $w$. When we alter the scenario still further, so that three students are involved ((29 iv)), (16) gets better still. And so on.

(29) Other scenarios

\begin{tabular}{|c|c|c|c|c|c|c|c|}
\hline M & $\mathrm{T}$ & W & $\mathrm{Th}_{\mathrm{h}}$ & F & $S$ & $\begin{array}{l}\text { \# propositions in } \Sigma \text { that } \\
\text { we need to verify to know } \\
\text { whether }(21) \text { is true }\end{array}$ & Judgment \\
\hline i. a & $a$ & $\mathrm{a}$ & $\mathrm{b}$ & b & $b$ & two & $? ? ?$ \\
\hline ii. a & $\mathrm{a}$ & $\mathrm{a}$ & $\mathrm{a}$ & a & b & two & $? ?$ \\
\hline iii. a & a & $\mathrm{a}$ & $b$ & $\mathrm{a}$ & $\mathrm{a}$ & two & $? ?$ \\
\hline jv. a & a & b & $\mathrm{b}$ & $c$ & c & three & $? ?$ \\
\hline v. a & a & $\mathrm{b}$ & $\mathrm{c}$ & $\mathrm{d}$ & $a$ & four & $?$ \\
\hline
\end{tabular}

\section{A constraint on the use of sentences with adverbial quantifiers}

One way of looking at these facts is as follows. A speaker who utters (16) in the scenarios that differ from our first scenario (on which a different student finished first each time) is quantifying over more things than he needs to in order to make his point. In using the sentence in (16), he is stating that all of the times in one set have a certain character - but he knows that we could have drawn this conclusion if he had informed us that all of the times in a smaller set had that character. Specifically, in using the sentence in (16) he is stating that all members of $\{$ Monday, .... Saturday $\}$ are such that the fastest student on that day was Swedish. But he knows that we could have drawn this conclusion if he had informed us that all of the times in a smaller set have this character. On our second scenario, one such smaller set is $\{$ Tuesday $\}$.

Conjecture: perhaps there is something wrong with quantifying over more things than you need to in order to make your point.

I think that judgments of the sentence in (30) reinforce this impression. The judgments of (30) are parallel to the judgments of (16). In the context of our first scenario, it seems a reasonable sentence for the speaker to utter in order to communicate something (negative) about the nationalities of the fastest students. In the context of our second scenario, it does not. On a plausible analysis of (30), a speaker who used it in this context would be claiming that none of the members of $\{$ Monday, ..., Saturday $\}$ are such that the fastest student on that day was Swedish. In the case of the second scenario, however, the speaker is aware that, to get the addressee to draw this conclusion, he could confine himself to the claim that none of the members of a smaller set - say $\{$ Tuesday \} - have the relevant property.

\section{The student who finished first was never Swedish.}

So I will follow through with the conjecture. I propose, roughly speaking, that a principle of pragmatics tells us not to use a sentence like (16) (or (30)) to quantify over the times in one set when we know that the hearer could draw the conclusion that the sentence is true by considering a parallel quantification over a smaller set. A sentence like (16) (or (30)) will sound funny if we think that the speaker who used it violated that principle.

To be more precise, the principle that I have in mind regulates the sentences that a speaker can make use of by regulating the $l f s$ that he can make use of. Given my assumptions so far, it is natural to suppose that a speaker who asserts a sentence has in mind a particular if for the sentence (call it L) and a particular assignment (call it $\mathrm{g}$ ), and that his purpose in uttering the sentence is (among other things) to convey that $[[\mathrm{L}]]^{\mathrm{g}}$ characterizes the actual 
world. Let's assume this. ${ }^{3}$ The principle that I have in mind narrows down what a speaker can hope to convey in this way by declaring some lfs, on some occasions, off limits. Some sentences have only one if, some have more than one. The principle will effectively ban a speaker from asserting a sentence if, for example, the sentence has only one If, and the principle blocks the use of this lf.

To talk about lfs, I will use the following (informal) terminology:

First of all, consider again the way an If with always gets interpreted.

$\left[\left[\text { always }_{i}[\alpha]\right]\right]^{g}=$

$\lambda w$. for all time intervals $t$ in the contextually salient set of time intervals, $[[\alpha]]^{g i->t}(w)=1$.

Informally speaking, lfs of the form [always $\mathrm{s}_{\mathrm{i}} \alpha$ ] quantify over members of the contextually salient set of time intervals. We might express this by calling this set the domain of quantification for $\left[\left[\text { always }_{\mathrm{i}} \alpha\right]\right]^{\mathrm{g}}$.

For every lf of the form [always $\mathrm{s}_{j} \alpha$ ] and assignment $\mathrm{g}$, we can imagine an alternative function that differs only with respect to what set is being quantified over.

a. $\lambda w$. for all time intervals $\mathrm{t}$ in set $\sigma 1,[[\alpha]]^{\mathrm{g} i->t}(\mathrm{w})=1$.

b. $\lambda w$. for all time intervals $t$ in set $\sigma 2,[[\alpha]]^{\mathrm{g} i>t}(w)=1$.

c. $\lambda w$. for all time intervals $t$ in set $\sigma 3,[[\alpha]]^{g i->t}(w)=1$.

Let us call these functions domain-variants of $\left[\left[\text { always }_{\mathrm{i}} \alpha\right]\right]^{\mathrm{g}}$. (32a) is a domain-variant of $[[$ always $\left._{i} \alpha\right]^{\mathrm{g}}$ with domain $\sigma 1,(32 \mathrm{~b})$ is a domain-variant of $\left[\left[\text { always }_{\mathrm{i}} \alpha\right]\right]^{\mathrm{g}}$ with domain $\sigma 2$, etc. Similarly, $\sigma 1$ is the domain for the domain-variant of [[ always $\alpha$ ] ] $]^{\mathrm{g}}$ given in (32a), etc. In general, lfs of the form [QUANT $i \alpha$ ] - where QUANT $_{i}$ stands for an adverbial quantifier -- will behave analogously to lfs of the form [always $s_{i} \alpha$ ]. That is, their denotations will be as in (33). Accordingly, we will be able to talk analogously about domain-variants of [[QUANT $i$ $\alpha]]^{\mathrm{g}}$.

$\left[\left[\text { QUANT }_{\mathrm{i}} \alpha\right]\right]^{\mathrm{g}}=$

$\lambda \mathrm{w}$. for proportion $\delta$ of the time intervals t in the contextually salient set of time intervals, $[[\alpha]]^{g i->t}(w)=1$.

With this in mind, here is a stab at the principle that constrains a speaker's choice of If. It is a rule for speakers to follow:

\footnotetext{
${ }^{3}$ In general, when people talk about the readings that sentences have, they might be presupposing something like this. One way of construing the claim that a sentence does/does not have a particular reading is as saying that a speaker can/cannot come up with a relevant If and assignment that together yield a particular kind of function from worlds to truth values. In Section 5, I too will talk about readings, I will assume that this is the right way of construing talk about readings, and moreover I will assume, together with a lot of literature, that people have intuitions about the readings that a sentence can have.
} 
(P) Rule:

(For any g),

Do not use an lf of the form [QUANT [Q $_{\mathrm{i}} \alpha$ to express that [[QUANT $\left.\left.\mathrm{T}_{\mathrm{i}} \alpha\right]\right]^{\mathrm{g}}$ holds of the actual world

when you can find a domain-variant of [[QUANT $\alpha]]^{\mathrm{g}}, \Delta$, with the following characteristics:

i. the domain for the domain-variant $\Delta$ is a proper subset of the domain for $\left[\right.$ QQUANT $\left.\left._{i} \alpha\right]\right]^{\mathrm{g}}$

ii. it follows from what the parties to conversation are taking for granted about the actual world that $\left[\left[{ }_{\text {QUANT }} \alpha\right]\right]^{\mathrm{g}}$ holds of the actual world as long as $\Delta$ holds of it. ${ }^{4}$

On its own, of course, this principle does not explain why sentences sound strange. Over and above this, I assume that a hearer can reflect on whether the assertion of a sentence obeys (P) or not, and will find the sentence strange if it does. Specifically, I propose (Q) (both aspects of which hopefully derive from more general aspects of the way we judge sentences).

(Q) a. A sentence whose only lf is of the form [QUANT $\mathrm{i} \alpha$ ] will sound strange if we think that the use of this lf (together with any assignment) violates $(\mathrm{P}) .^{5}$

b. If we know that the speaker can find a $\Delta$ that has the relevant characteristics and that has only one element in its domain, then the sentence will sound terrible. Less terrible if two, etc.

Now here is how principle (P) will apply to the use of (16) on our second scenario. By assumption, (16) has only one kind of If - the one given in (17) - and the denotation of this lf with respect to an arbitrary assignment $\mathrm{g}$ is repeated in $(34 \mathrm{a}, \mathrm{b})$. (The denotation will be the same no matter what assignment the speaker chooses. In what follows I will be sloppy and write $[[(17)]]^{g}$ to mean this one object that is the denotation of (17) with respect to any assignment. This might cause some confusion but I trust the reader to correct for it.)
a. $[[(17)]]^{\mathrm{g}}=\lambda \mathrm{w}$. For all days $\mathrm{t}$ in $\mathrm{S}$, the individual who in $\mathrm{w}$ is the student first to finish the exam held in $t$ is Swedish-born in $w$.
b. $S=\{$ Monday, Tuesday, Wednesday, Thursday, Friday, Saturday $\}$

One possible domain-variant $\Delta$ of $[[(17)]]^{\mathrm{g}}$ is the one given in (35).

One possible domain-variant:

$\Delta=\lambda \mathrm{w}$. For all days $\mathrm{t}$ in \{Tuesday\}, the individual who in $\mathrm{w}$ is the student first to finish the exam held in $t$ is Swedish-born in $w$.

( $=\lambda \mathrm{w}$. The individual who in $w$ is the student first to finish the exam held on Tuesday is Swedish-born in w. )

It so happens that the domain of $\Delta$ is $\{$ Tuesday $\}$, which is a proper subset of the domain of

\footnotetext{
${ }^{4}$ Call $C$ the set of worlds compatible with the information that the parties to conversation are taking for granted (cf. Stalnaker 1979). Then another way of putting (ii) is as follows: For every world $w$ in $C,\left[\left[Q U A N T_{i} \alpha\right]\right]^{g}(w)$ $=\mathrm{I}$ iff $\Delta(\mathrm{w})=1$.

${ }^{5}$ More precisely: if, given what we think about what the speaker thinks is being taken granted about the actual world, it follows that the speaker would violate $(\mathrm{P})$ by using the relevant lf.
} 
$[[(17)]]^{g}$. What this means given (P) is that, if it follows from what the parties to conversation are taking for granted about the actual world that $[[(17)]]^{\mathrm{g}}$ holds of the actual world as long as $\Delta$ does, then the speaker is banned from using (17).

Now, on our second scenario, this does plausibly follow from what the parties to conversation are taking for granted. The important aspect of this scenario was that both parties to conversation were present at all the exams and saw that the same student finished first each time. This makes it likely that the following is being taken for granted:

(36) For all $t 1, t 2$ in $S$, the individual who in w0 is the student first to finish the exam in $\mathrm{t} 1$ is the individual who in w0 is the student first to finish the exam in $t 2$.

But given (36), if $\Delta(\mathrm{w} 0)=1$ we can conclude that $[[(17)]]^{\mathrm{g}}(\mathrm{w} 0)=1$ and if $\Delta(\mathrm{w} 0)$ is not 1 we can conclude that $[[(17)]]^{\mathrm{g}}$ (w0) is not 1 . (We went through the relevant reasoning in the previous section.) In other words, given (36) we can conclude that $[[(17)]]^{\mathrm{g}}(\mathrm{w} 0)=1$ as long as $\Delta(w 0)=1$. So - assuming (36) is indeed being taken for granted $-(\mathrm{P})$ will prohibit the use of If (17) on the second scenario. Moreover, since by assumption (17) is the only lf that the sentence in (16) has, (P) will effectively prohibit the use of the sentence in (16).

What will be the consequence? If we think that (36) is being taken for granted (or more precisely that the speaker thinks that it is), then we will think that the speaker is violating $(\mathrm{P})$ by using (16). Accordingly, the utterance of (16) will sound strange. And given that the relevant domain-variant had only one element in its domain, the utterance of (16) will sound very strange.

In what follows, in discussing examples like these, I will sometimes take an expository shortcut: I will draw a diagram that represents the knowledge of the world that the parties to conversation are taking for granted. In the case of the scenario we just considered, the diagram would look like this:

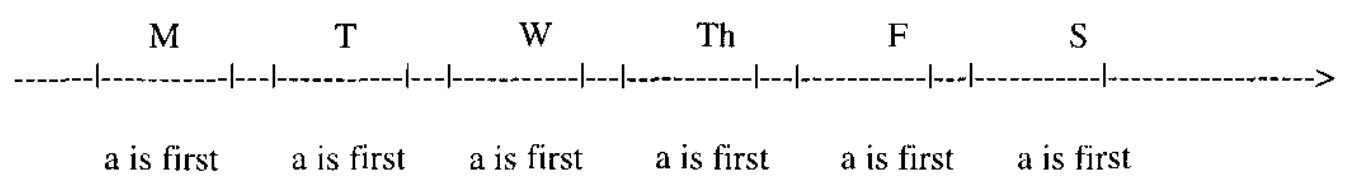

I will only be considering cases of lfs whose denotations are the same with respect to all assignments. To show that (P) blocks a particular lf, I will show that: (i) if we add to the diagram the information that a certain domain-variant characterizes the actual world, then from the information on the diagram we can conclude that the denotation of the lf characterizes the actual world; if we add to the diagram the information that the domainvariant does not characterize the actual world, then from the information on the diagram we can conclude that the denotation of the lf does not characterize the actual world. For example, if we add to the diagram the information that $\Delta$ characterizes the actual world 


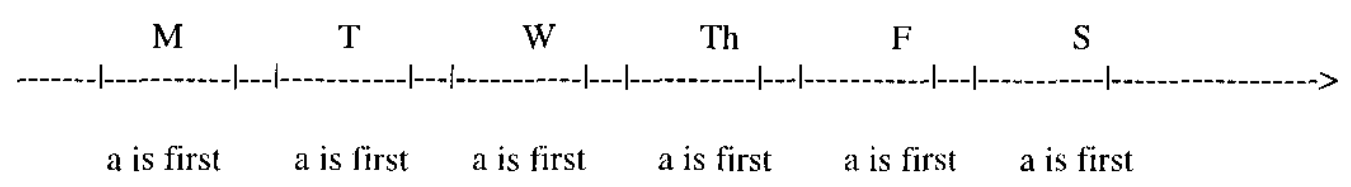

$\mathbf{a}$ is Swedish-born

we have enough information in the diagram to conclude that $[[(17)]]^{g}$ characterizes the actual world. If instead we add to the diagram the information that $\Delta$ does not characterize the actual world

b.

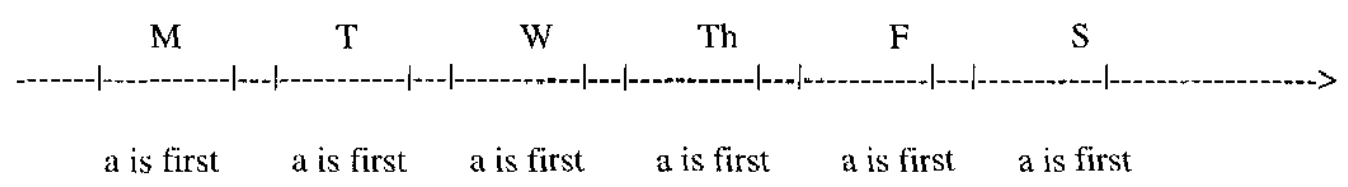

$a$ is not Swedish-born

we have enough information in the diagram to conclude that $[[(17)]]^{g}$ doesn't either.

\section{Some consequences of this constraint}

I will now demonstrate some of the consequences of the pragmatic constraint that I have identified. I am going to go through a number of sentences which have Ifs of the form [QUANT $\mathrm{i}_{\mathrm{i}} \alpha$ ], and see what it predictions it makes about the felicity of these sentences.

If the approach that I have been taking is correct, then there are a number of factors that play a role in determining the infelicity of a sentence with an If of the form [QUANT $\alpha$ ]. One is the denotation of $\alpha$, because that will play a role in determining the denotation of the whole If and therefore its possible domain variants. Another is the specific set of times that the context makes salient, because that is what functions as the domain of [[QUANT $\alpha]]^{\mathrm{g}}$. Another is what we think is being taken for granted about the world, since this will play a role in determining what we can conclude about the truth of the original sentence given the truth of a domain variant. So demonstrating the predictions that (P) makes will potentially require us to take a stand on all of these factors. We will see, however, that in a large number of cases, just taking a position on one or two of these factors will be enough to make a prediction.

One remark in advance. In examining the predictions that we make once we take $(P)$ into account, I am going to assume that apart from $(\mathrm{P})$ there is an additional constraint on the use of quantifiers like always and never:

Only use [ QUANT $\alpha$ ] if (for some g) the domain of quantification for $\left[\left[\text { QUANT }_{i} \alpha\right]\right]^{\mathrm{g}}$ contains more than three objects.

This can be independently motivated, and should follow from more general restrictions on the use of quantifiers (parallel restrictions apply to every, etc.). (Cf. de Hoop/de Swart 1989.) Without going through the reasoning, I will just note that some evidence for (39) comes from judgments of our old sentence $(16)(=(40 \mathrm{a})$ ) on some variants of our first scenario. On a 
scenario where there are only two exam days instead of six ((40b)), the sentence sounds very odd. When there are three $((40 \mathrm{c}))$, it still sounds odd (though less odd). When there are four $((40 d))$, it doesn't sound bad at all.

(40) a. The student who finished first was always Swedish.
b. $\mathrm{M}$ T ???
a b
c. $M$ T W ?
d. M T W Th not bad
a $\quad b \quad c$
a $\quad b \quad c \quad d$

\section{1}

The first case that I will consider is the case of the sentence we started out with ((41a)). I will assume that the only Ifs it has are of the kind we have considered so far, one where always combines with another constituent $((41 b))$.
a. Ingrid was always Swedish.
b. If: always ${ }_{i} \alpha[\ldots]$

What we assume to be the denotation of this other constituent will obviously play a role in when we predict the If in ( $41 \mathrm{~b})$, and thus the sentence in (41a), to be usable. I will assume the following about the constituent that always attaches to. ${ }^{6}$ I will assume that the constituent contains an index but the only purpose of this index is to restrict the domain of the function that we get out of this constituent. (This assumption isn't innocuous, and we will see that the contribution of the index plays a role in our reasoning about when (41a) will be felicitous.) The denotation of this constituent with respect to an assignment will be specifically as in $(42 b)$.
a. always $\alpha_{\mathbf{i}}[\ldots \mathbf{i}]$
b. $[[\alpha]]^{g}=$
$\lambda w$ : Ingrid is alive in $w$ for the duration of $g(i)$. Ingrid is Swedish-born in w. (cf. Musan 1995)

The result is that the If of (41a) will have a denotation as in (43), where $S$ is the contextually salient set of time intervals,

(43) $[[(41 b)]]^{g}(w)=1$ as long as for all $t$ in $S$, Ingrid is alive in $w$ for the duration of $t$ and Ingrid is Swedish-born in w.

and accordingly domain-variants of $[[(41 b)]]^{g}$ will be of the form

$$
\begin{aligned}
& \Delta(w)=1 \text { as long as for all } t \text { in } \sigma, \text { Ingrid is alive in } w \text { for the duration of } t \\
& \text { and Ingrid is Swedish-born in } w .
\end{aligned}
$$

Given this, we can derive (by making an additional assumption) that, no matter what exactly the salient time intervals are, a speaker is not permitted to utter (4la). This in turn means that a sentence like (41a) should always sound bizarre. Since it is hard to imagine an utterance of (41a) that sounds sensible, this prediction seems right.

\footnotetext{
${ }^{6}$ This can be seen as a minor change from what I have assumed so far in talking about The student who finished first was always Swedish, but it is not a change that affects the preceding discussion in any serious way.
} 
In brief, the reasoning is as follows. Lfs of the kind in (41b) are the only ones that (41a) admits, so if a speaker is prevented from using those he can't use (41a). Now, I assume that an independent principle of grammar (I will elaborate briefly below) guarantees that a speaker is only allowed to use (41b) when it is already established that Ingrid is alive at all the intervals in the contextually salient set. But if it is already established that Ingrid is alive at all the intervals in the contextually salient set (and there is more than one interval in this set), a speaker who uses $(41 \mathrm{~b})$ will violate $(\mathrm{P})$. To see this, imagine first the information that the parties to conversation are taking for granted:

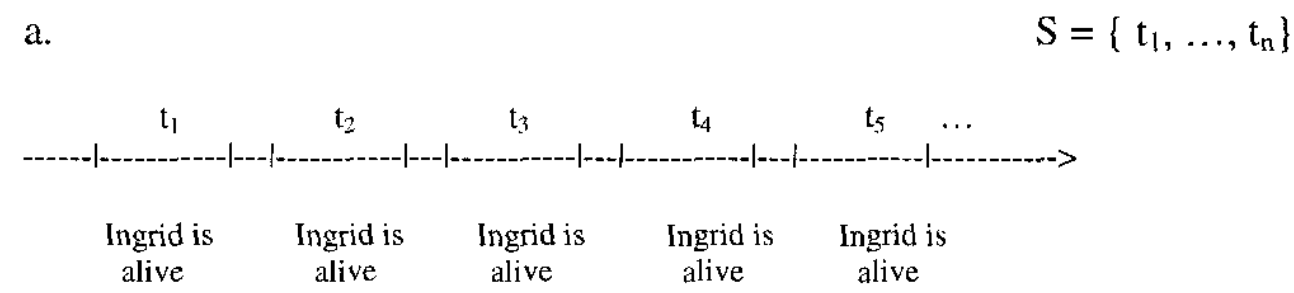

Now consider the domain-variant of $[[(41 \mathrm{~b})]]^{\mathrm{g}}$ whose domain consists only of $\mathrm{t}_{1}$, the first time interval in the contextually salient set. (Call it $\Delta$ again.) $\Delta$ characterizes a world $w$ as long as for all $\mathrm{t}$ in $\left\{t_{1}\right\}$, Ingrid is alive in $\mathrm{w}$ for the duration of $\mathrm{t}$ and Ingrid is Swedish-born in $\mathrm{w}$. That is, it characterizes a world $w$ as long as Ingrid is alive in $w$ for the duration of $t_{1}$ and Ingrid is Swedish-born in w. Suppose we add to the diagram the information that $\Delta$ characterizes the actual world. This amounts to adding:

\section{b. Ingrid is Swedish-born}

and now from the information in the diagram we can conclude that $[[(41 b)]]^{g}$ characterizes the actual world. Suppose instead we add to the diagram that $\Delta$ doesn't characterize the actual world. The only way of doing this consistently with the information already there is to add

\section{Ingrid is not Swedish-born}

and from this we can conclude that $[[(41 b)]]^{g}$ does not characterize the actual world.

(How about if there is only one interval in the contextually salient set? In this case, we will not be able to find a relevant domain variant of $[[(41 \mathrm{~b})]]^{\mathrm{g}}$ whose domain is a proper subset of $[[(41 \mathrm{~b})]]^{\mathrm{g}}$ 's domain, so (P) will not be violated. But the principle in (39) will be, so even in this case the speaker will be prevented from using the lf in (41b).)

The independent principle of grammar that I made use of in the course of my reasoning here is one that has been identified in discussions of "presupposition projection" (e.g. Heim 1983). ${ }^{7}$ Given the domain condition in the denotation of the sister of always (the denotation given in (42b)), this principle limits the conditions under which a speaker can use an If that contains this constituent. Specifically, if the If is one where this constituent combines with a quantifier (a quantifier coindexed with the index in this constituent), the speaker can only use the If when it is being taken for granted that a certain property holds of every item in the set the quantifier ranges over. Without going into detail, in this case, where

\footnotetext{
${ }^{7}$ One formulation that will do for the purposes here is: Do not use [ QUANT $\alpha$ ] to express that [[ QUANT $\alpha]]^{\xi}$ holds of w0 unless it is taken for granted about w0 that, for every $\mathrm{x}$ in the domain of [[ QUANT $\left.\left.\alpha\right]\right]^{\mathrm{g}}$, $[[\alpha]]^{\mathrm{g} i \mathrm{x} \times}(w 0)$ is defined. See Percus 1998 for a version of the principle that is very close to this one.
} 
the quantifier ranges over time intervals, every one of these time intervals must be one during which Ingrid is alive.

(45) Consequence of one view of "presupposition projection"and (42b):

A speaker is entitled to use (41b) to express that [[ (41b) $]]^{g}$ holds of w0 only when (46) is taken for granted.

(46) For every $t$ in the domain of quantification for $[[(41 b)]]^{g}$, Ingrid is alive in w0 for the duration of $\mathrm{t}$.

\section{2}

The next case I want to consider is (47). Here as before, I will assume that there is just one possible kind of lf, one in which always attaches to another constituent, and that this constituent is interpreted as in $(48 b)$.

Napoleon was always dead.

a. always $_{\mathbf{i}} \alpha[\ldots \mathbf{i}]$

b. $[[\alpha]]^{g}=\lambda w$. Napoleon is dead in $w$ for the duration of $g(i)$.

This means that the If of (47) will have a denotation as in (49), where $S$ is the contextually salient set of time intervals.

$$
[[(48 a)]]^{g}=\lambda w \text {. for all } t \text { in } S, \text { Napoleon is dead in } w \text { for the duration of } t
$$

and accordingly domain-variants of $[[(48 \mathrm{a})]]^{\mathrm{g}}$ will be of the form $\lambda w$. for all $t$ in $\sigma$, Napoleon is dead in $w$ for the duration of $t$.

The fact is that, as with the previous sentence, it is hard to imagine an utterance of (47) that does not sound bizarre. My feeling is that when we try to imagine a speaker uttering the sentence, we have the impression that the speaker who utters it expects that at any moment Napoleon could have come back to life, as in "I kept checking the coffin, but Napoleon was always dead." That a speaker should expect something like this itself seems bizarre.

What we can derive on these assumptions about the syntax and semantics of (47) is that (47) will be unusable when it is taken for granted that anyone who is dead at one point is dead at all later points $((50))$ - again, irrespective of what exactly those time intervals are that the context makes salient. This is because, no matter what set of intervals the context makes salient, a speaker who uses the If in (48a) while (50) is taken for granted will violate (P). Since lfs of the kind in (48a) are the only ones that (47) admits, as long as (50) is taken for granted, a speaker will never be able to felicitously utter (47).

(50) Fact about the actual world w0:

For all $\mathrm{x}, \mathrm{t}, \mathrm{t}$, , if $\mathrm{x}$ is dead in $\mathrm{w} 0$ at $\mathrm{t}$ and $\mathrm{t}$ ' contains no moment that precedes $\mathrm{t}$ then $\mathrm{x}$ is dead in w0 at $\mathrm{t}$.

To see this, again imagine the information that is being taken for granted: 
a.

$$
S=\left\{t_{1}, \ldots, t_{n}\right\}
$$

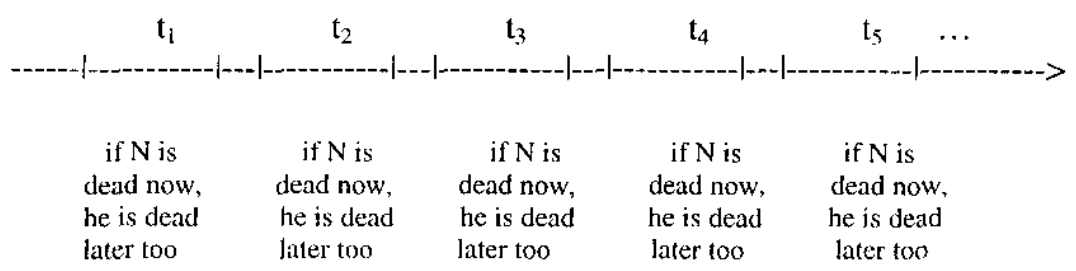

Now consider the domain-variant (call it $\Delta$ again) whose domain consists only of $t_{1}$, the first time interval in the contextually salient set. Suppose we add to the diagram the information that $\Delta$ characterizes the actual world:

b.

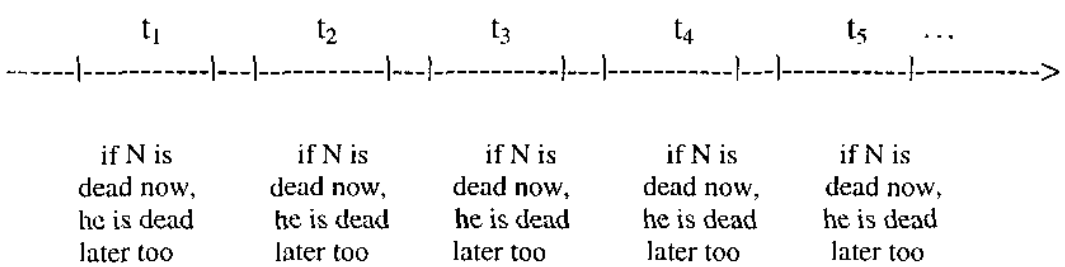

$\mathbf{N}$ is dead

now

From this we can conclude that $[[(48 \mathrm{a})]]^{\mathrm{g}}$ characterizes the actual world. Suppose instead we add the information that $\Delta$ does not characterize the actual world:

c.

$\begin{array}{ccccc} & t_{1} & t_{3} & t_{4} & t_{5} \\ \text { if } \mathrm{N} \text { is } & \text { if } \mathrm{N} \text { is } & \text { if } \mathrm{N} \text { is } & \text { if } \mathrm{N} \text { is } & \text { if } \mathrm{N} \text { is } \\ \text { dead now, dead now, } & \text { dead now, dead now, dead now, } \\ \text { he is dead } & \text { he is dead } & \text { he is dead } & \text { he is dead } & \text { he is dead } \\ \text { later too } & \text { later too } & \text { later too } & \text { later too } & \text { later too }\end{array}$

$\mathbf{N}$ is not

dead now

From this we can conclude that $[[(48 \mathrm{a})]]^{\mathrm{g}}$ does not characterize the actual world.

Here as in the previous case, the conclusion that (47) is unusable depends in part on the idea that the parties to conversation are accepting certain assumptions about the world. The relevant assumption here is that once you are dead, you're dead. Unlike in the previous case, however, there is no principle that links the acceptance of this assumption to the fact that the speaker has decided to use (47). Therefore, we predict that in cases where it is clear that the parties to conversation are not making this assumption, the sentence might not seem strange. Although we generally do accept that once you are dead, you're dead, stories about resurrection or about supernatural beings that come back to life suggest that one might choose not to accept this. Here is a case parallel to (47) in which the speaker specifically disavows the assumption that once you are dead, you're dead. The relevant clause here does not sound so bizarre, so this at least is consistent with the story we have told. 
Vampires are supposed to come back to life after midnight, but I checked the coffin every half hour and I can assure you that this vampire was always dead.

Here is a minimal pair that suggests that the story of the kind here is on the right track. It doesn't involve dead but rather tall and blue-eyed. A parallel account of (53) would yield roughly the following: if the parties to conversation presume that once you are tall and blueeyed, you are tall and blue-eyed for good, then (53) will lead to a violation of (P). This is the kind of assumption that we might normally make, but not in a context where the speaker acknowledges the existence of supernatural forces, like witches' spells, that can induce shapechanging. (55) is a case where the speaker acknowledges the existence of such forces; (54) is a case where he doesn't. My impression is indeed that in (54) but not in (55), the last sentence comes as a bit of a surprise. It seems to suggest that the medication could have changed John's height or eye color, and this is not what we normally expect medication to be able to do.

(53) John was always tall and blue-eyed.

(54) The experimenters gave them a new medication every Tuesday evening. The next morning, the two of them generally looked in the mirror to see if it had had any effect on them physically. Ingrid went through quite a variety of changes over the weeks, without much of a common denominator. One week she would find that she was more muscular, the next week she would find that she was a little wrinkly. John, on the other hand, was always tall and blue-eyed.

(55) The witch cast a spell on them every Tuesday evening. The next morning, the two of them generally looked in the mirror to see what changes she had made to their appearance. Ingrid went through quite a variety of changes over the weeks, without much of a common denominator. One week she would find that she was large and muscular, the next week she would find that she was frail and wrinkly. John, on the other hand, was always tall and blue-eyed.

A variant of this case that is worth considering is the example in (56) - I assume that as before the only kind of If that the sentence has looks like (57a), that the denotation of this If is as in (58) and that accordingly domain-variants will be of the form $\lambda \mathrm{w}$. for all $t$ in $\sigma$, Napoleon is alive in $w$ for the duration of $t$.

Napoleon was always alive.

a. always $\mathbf{i}_{\alpha}[\ldots \mathbf{i}]$

b. $[[\alpha]]^{g}=\lambda w$. Napoleon is alive in $w$ for the duration of $g(i)$.

$[[(57 \mathrm{a})]]^{\mathrm{g}}=\lambda \mathrm{w}$. for all $\mathrm{t}$ in $\mathrm{S}$, Napoleon is alive in $\mathrm{w}$ for the duration of $\mathrm{t}$

Here we again derive that the sentence will be unusable when the speaker is taking for granted that anyone who is dead at one point is dead at all later points -- irrespective of what exactly those time intervals are that the context makes salient. However, this example is slightly different from the earlier one. In the absence of accepted knowledge about whether Napoleon was born yet at the time of the first interval, the kind of domain-variant that we need to consider in order to demonstrate a $(\mathrm{P})$ violation is different from the kind we needed to consider earlier. It is one with a larger domain. Specifically, its domain must consist of two 
intervals: the first interval and the last interval of the contextually salient set. (I leave it to the reader to verify this.) Now, the bizarreness of our old example (47) when judged in the absence of context suggests that in the absence of context we seem by default to take it for granted that once you are dead you're dead. So, given the proposal thus far, one might expect that this sentence too will sound bizarre when judged in the absence of context - but perhaps a little less bizarre. That seems to be the prediction.

\section{3}

In the case of the sentences we just looked at, anytime the parties to conversation are taking for granted the kind of assumptions that we all generally make about the world - for instance, that once you're dead you're dead - the speaker will violate $(\mathrm{P})$. And this is true irrespective of what set of time intervals it is that the context makes salient. Accordingly, as Iong as we have no reason to think that the parties to conversation are departing from these normal assumptions, ${ }^{8}$ we don't need to make any additional commitments as to what the salient set of time intervals is in order to reject the sentence as bizarre. At least, that is what follows from the picture I have presented so far.

The case of $(61)(=(5))$ is different. (I will assume as before that $(61)$ has just one kind of lf - given in (62a) - and that the denotation of this If is as in (63).)

(61) John always knows whether Ingrid is Swedish.

(62) a. always $\mathbf{s}_{\mathbf{i}}[\ldots \mathbf{i}]$

b. $[[\alpha]]^{\mathrm{g}}=\lambda \mathrm{w}$. in $\mathrm{w}$, John knows for the duration of $\mathrm{g}(\mathrm{i})$ whether Ingrid is Swedish ${ }^{9}$

$[[(62 a)]]^{g}=\lambda w$. for all $t$ in $S$, in $w$ John knows for the duration of $t$ whether Ingrid is Swedish

We would be able to say the same thing about (61) if we could maintain, for instance, that a normal assumption that we make about the world is that, once you know whether Ingrid is Swedish, you retain that knowledge forever. But I think that is not the kind of assumption that we normally make. We normally imagine that knowledge of this kind tends to fade, especially if you are never called upon to access it. In this case, if indeed the sentence sounds bizarre to us, that must have something to do with assumptions we are making about the set of time intervals that is being quantified over.

Here is the kind of situation in which we predict that a speaker who uses (61) will violate $(\mathrm{P})$. We predict a violation of $(\mathrm{P})$ when it is taken for granted that the distance between intervals is smaller than the amount of time that it would typically take for John's memory to fade. The idea is this: suppose it is taken for granted that, if John knows at interval $\mathrm{t}_{2}$ whether Ingrid is Swedish, then he knows this also at the succeeding interval $t_{3}$.

\footnotetext{
${ }^{8}$ Or more precisely: as long as we have no reason to think that the speaker is assuming this kind of departure from normal assumptions.

${ }^{9}$ More precisely, to be consistent with the assumptions so far: Swedish-born.
} 


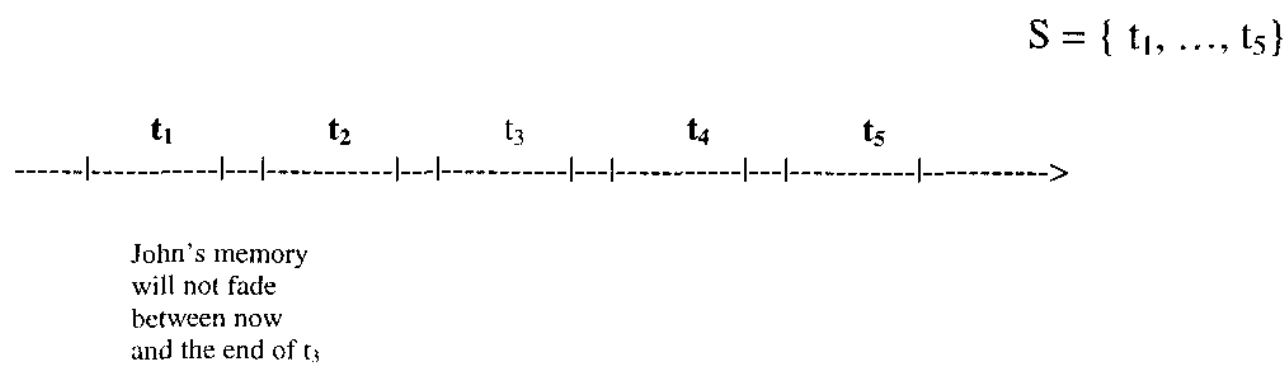

Then to determine whether he knows at all of the intervals in $S$ whether Ingrid is Swedish, it is enough to determine whether he knows at all the intervals except $t_{3}$ whether Ingrid is Swedish. Consequently, we can show that the use of the lf (62a) violates $(\mathrm{P})$ by considering a domain variant of $[[(62 a)]]^{g}$ whose domain consists of all the intervals except $t_{3}$.

What this means is that, to the extent that we feel that the sentence is bizarre when we have no clue as to what the relevant time intervals are, we must be making some additional assumption along these lines: the distance between intervals is smaller than the amount it would take for John's memory to fade.$^{10}$ On the approach that I have been taking, this is the conclusion we are led to.

To test whether the approach is on the right track, what we should do is consider two kinds of scenarios, one where it is clearly taken for granted that the distance between intervals is smaller than the amount of time it takes for John's memory to fade, and one where it is clearly taken for granted that it is larger. A sentence like (61) should seem odd in the first scenario, but all right in the second. In fact, it is hard to think of any scenario where the kind of information that (61) conveys might be of interest, but the contrast between the following two dialogues might bear out the prediction. I think that the final sentence of the first dialogue sounds a lot worse than the final sentence of the second dialogue (where a natural paraphrase would use "remember" instead of "know"). When we hear the first dialogue, our first impression is that "always" is just redundant. If we try to justify it to ourselves, then if anything we have the strange feeling that the speaker thinks that the relevant information might suddenly vanish from John's mind, and when we know nothing else about John this comes as a surprise.

(65) -- I heard that Ingrid is Scandinavian, and I wanted to know whether she is Swedish.

-- For some reason this question comes up a lot. I always send people to John to find out. He isn't very knowledgeable, it's true, but he does always know whether Ingrid is Swedish.

(66) -- I heard that Ingrid is Scandinavian, and I wanted to know whether she is Swedish.

-- For some reason this question comes up a lot. I always send people to John to find out. He has a lousy memory for many things, but he does always know whether Ingrid is Swedish.

(A more realistic minimal pair might be as in (67)-(68), which I think behave similarly.)

(67) -- I was advised to buy a box of Fenistil, but I need to know first whether it contains antihistamines.

\footnotetext{
${ }^{10}$ Or more precisely: that the speaker is taking this for granted.
} 
-- For some reason this question comes up a lot. Why don't you go ask the assistant pharmacist? He isn't very knowledgeable, it's true, but he does always know whether Fenistil contains antihistamines.

(68) -- I was advised to buy a box of Fenistil, but I need to know first whether it contains antihistamines.

-- For some reason this question comes up a lot. Why don't you go ask the assistant pharmacist? He has a lousy memory, it's true, but he does always know whether Fenistil contains antihistamines.

\section{Consequences for "semantic partition"}

Here is a very general pattern of reasoning. It starts from the claim that we do not use a sentence $\mathrm{S}$ to express the proposition that would be derived from an $1 \mathrm{~L}^{11}{ }^{11}$ It concludes on this basis that $L$ is not a possible If for a sentence $S$.

Now that we have seen that principles of pragmatics can prevent us from using an If, we can see that this kind of reasoning is questionable. If we do not use a sentence to express the proposition that would be derived from an If $\mathrm{L}$, that could be because other principles prevent us from using L. It doesn't have to be because our mechanism for generating lfs prevents us from generating $\mathrm{L}$ for the sentence in question.

One place where this questionable pattern of reasoning has been used is in the treatment of sentences like Ingrid was always Swedish. We saw that there an independent principle of pragmatics, $(\mathrm{P})$, could explain why we do not use an If that contains an item coindexed with the quantifier. I want now to look at another case where this pattern of reasoning has been used, and ask if instead of saying that the sentence lacks an If that we might otherwise expect, we can again say that while in principle we can generate this If, in practice $(\mathrm{P})$ will typically prevent us from using it.

The sentences that I am interested in are sentences with "individual-level predicates," and I am interested in the way they behave with respect to "semantic partition." Discussions of the syntax and semantics of sentences with singular indefinite subjects often start with the idea that sentences like (69) have among their possible lfs one that gives rise to a proposition like (70). They propose what that if is and then argue that the parallel if is not available for sentences like $(71)$.

(69) A secretary is always on the phone.

(70) $\lambda \mathrm{w}$. For all intervals $t$ in the set of intervals that the context makes salient, there is some individual who in $\mathrm{w}$ is a secretary for the duration of $\mathrm{t}$ and who in $\mathrm{w}$ is on the phone for the duration of $\mathrm{t}$

(71) A secretary is always Swedish.

The argument follows the pattern of reasoning that I just sketched: We do not use a sentence like this to express the proposition that we would derive from the relevant If. Therefore, the sentence does not have the relevant lf.

\footnotetext{
${ }^{11} \mathrm{I}$ am using proposition here to mean a function from worlds to truth values. I am using express proposition $p$ to mean "convey that $p$ characterizes the actual world." See Section 3.
} 
The kind of Ifs that we are interested in are lfs like those in (72) and (73), or minor variants of these.

(72) always 1 [vp [DP a $a_{2}$ secretary $\left.e_{1}\right]\left[t_{2}\right.$ on the phone $\left.\left.e_{I}\right]\right]$

(73) always $_{1}\left[\mathrm{vP}\left[\mathrm{DP} \mathrm{a}_{2}\right.\right.$ secretary $\left.\mathrm{e}_{1}\right]\left[\begin{array}{lll}\mathrm{t}_{2} & \text { Swedish } \mathrm{e}_{1}\end{array}\right]$

I have sketched in (74)-(76) a few aspects of the way these Ifs are interpreted. Some things that are worth noting are that the indexed $e$ items are silent items that function as variables over times, and that the DP functions as a quantificational expression with existential force.

$\left[\left[\quad\left[\begin{array}{lll}{[D p} & \left.a_{i} \text { secretary } e_{j}\right] & \alpha\end{array}\right] \quad\right]\right]^{g}$

$=\lambda \mathrm{w}$. there is some individual $\mathrm{x}$

such that, in $w, x$ is a secretary for the duration of $g(j)$ and such that $[[\alpha]]^{\mathrm{g} i-\mathrm{x}}(\mathrm{w})=1$.

(75) $\left[\left[\mathrm{VP}_{(72)}\right]\right]^{\mathrm{g}}=\lambda \mathrm{w}$. there is some individual $\mathrm{x}$

such that, in $w, x$ is a secretary for the duration of $g(1)$ and such that, in $w, x$ is on the phone for the duration of $g(1)$

(76) $\left[\left[\mathrm{VP}_{(73)}\right]\right]^{\mathrm{g}}=\lambda \mathrm{w}$. there is some individual $\mathrm{x}$ such that, in $w, x$ is a secretary for the duration of $g(1)$ and such that $\mathrm{x}$ is Swedish-born in $\mathrm{w}$

The propositions that we would get out of these Ifs are what I have written in (77) and (78). The If in (72) would say that we can find a secretary on the phone at each of the contextually salient time intervals; the parallel If in (73) would say that we can find a Swedish secretary at each of the contextually salient time intervals. The position I am examining has it that, while we can generate the If in (72) for the sentence A secretary is always on the phone, we cannot generate the parallel if in (73) for the sentence $A$ secretary is always Swedish. Is this position justified?

$[[(72)]]^{\mathrm{g}}=$

$\lambda w$. For all intervals $t$ in the set of intervals that the context makes salient, there is some individual who in $w$ is a secretary for the duration of $t$ and who in $w$ is on the phone for the duration of $t$

$[[(73)]]^{\mathrm{g}}=$

$\lambda w$. For all intervals $t$ in the set of intervals that the context makes salient, there is some individual who in $w$ is a secretary for the duration of $t$ and who is Swedish-born in w

The point that I want to make is that, even if A secretary is always Swedish does have the If in (73), the use of this If will be very restricted. As long as we take it for granted that at two of the contextually salient time intervals exactly the same people are secretaries, $(\mathrm{P})$ will prevent us from using it. Suppose for instance that it is accepted that the pool of secretaries is the same from, say, $t_{2}$ to the end of $t_{3}$; then $(P)$ will prevent a speaker from using (73) by virtue of the domain-variant whose domain simply excludes $t_{3}$. This is because, to determine whether there is a Swedish-born secretary at each of the relevant intervals, it is enough to 
determine whether there is a Swedish-born secretary at every interval but $t_{3}$. (By contrast, to determine whether there is a Swedish-born secretary on the phone at each of the relevant intervals, it is not enough to determine whether there is a Swedish-born secretary on the phone at every interval but $t_{3}$. So $(P)$ will not prevent a speaker from using (72) in the same situation.)

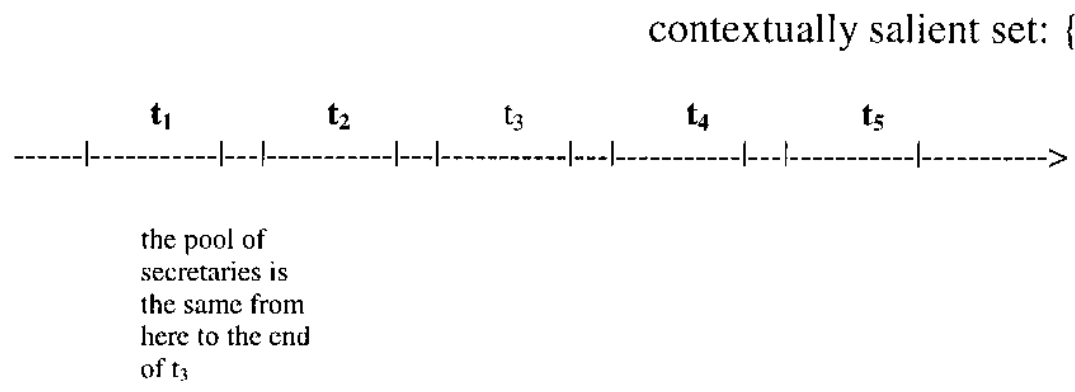

In fact, if the contextually salient time intervals are close together, it is pretty reasonable to imagine that the pool of secretaries will stay the same from one time interval to the next.

What does this mean? It means that, to the extent that we do not naturally take the sentence to express the proposition in (78) in the absence of information about the relevant time intervals, this might not be because the sentence lacks the If in (73). It could just be because, in the absence of contrary information, we tend to imagine that the pool of secretaries will stay the same from one interval to the next - maybe because we tend to imagine that the intervals are close together. This kind of default assumption would be analogous to the kind of default assumption we considered at the end of the last section: namely that, in the absence of contrary information, we imagine that knowledge of whether Ingrid is Swedish will stay present from one interval to the next. If we really want to know whether the If in (73) is a possible one, we should at least make sure to look at cases where it is clear that changes in the secretary pool occur between the contextually salient intervals, and cases like these will have to have intervals that are fairly far apart. A candidate case is (80). (To judge by the nods at the workshop,) it seems that (80) can convey that every change of office staff results in the inclusion of a Swede in the secretary pool, and this suggests that the If in (73) is possible. ${ }^{12,13}$

\footnotetext{
${ }^{12}$ I think that there is an additional aspect of $(80)$ that contributes to bringing out the reading I am interested in. This is the contrast between secretary and technical assistant. (On the natural way of pronouncing (80), there is pitch accent on both technical and secretary.) A near minimal pair (for me) that suggests that the contrast makes a difference is (i). The continuation in (a) is bizarre, suggesting that none of its possible lfs is appropriate. By contrast, the continuation in (b) is good and conveys that most staff reviews turn up a Swedish secretary.

(i) We require them to hire Swedes for as many positions as possible. With this in mind, we review their staff every year. In fact,

a. ?? a secretary is usually Swedish.

b. none of the technical assistants are ever Swedish. Still, a SEcretary usually is.

Significantly, I think, for the analysis of this contrast, replacing "a secretary" in (i a) by "one of the secretaries" redeems the sentence.

${ }^{13}$ One might imagine that the lfs for sentences like Some secretary is always Swedish or At least one of the secretaries is always Swedish yield propositions just like the one (73) yields. Suppose they do. I have suggested here that, in the case of (73), assumptions that we make in the absence of contrary information prevent us from using lfs like (73). Does this imply that these other sentences should sound odd in the absence of special information? The prediction here depends on other factors. When we are exposed to a sentence and find that $(\mathrm{P})$ taken together with our natural assumptions rules out one of its lfs, we have various options other than rejecting the sentence. If the sentence has another If that yields a different proposition, and that does not violate $(\mathrm{P})$, we could decide to maintain our natural assumptions and to take the sentence as expressing that different proposition. Or we could decide to revise our assumptions. (In the case of sentences like (71), it has been
} 
(80) Every five years, we change office staff entirely. We never wind up with any Scandinavians as technical assistants, but by sheer chance a secretary is always Swedish.

If this line of reasoning is right, there are still some questions to ask. But they have nothing to do with the lfs that (71) admits. The main question is why, when we ask ourselves whether it gives rise to the reading in (78), we have a strong tendency to imagine that the times being quantified over are close together rather than far apart. We seem not to exhibit this tendency, for example, when we ask ourselves what a sentence like (81) expresses. Here, we seem to imagine that there is only one relevant time interval per reign. ${ }^{14}$

A blond is always King of Sweden, and a brunette is always Queen.

I want to close this section by mentioning a prediction that we can now make. I just said with regard to sentences like (71) that, when we have to guess what kind of time intervals might be under consideration, unless we know otherwise, we apparently do not imagine that changes in the secretary pool occur between the intervals. That is the conclusion we have to draw if (71) allows the If in (73). As we saw earlier, we can draw a parallel conclusion from the fact that a sentence like John always knows whether Ingrid is Swedish sounds bizarre in the absence of information about the time intervals under consideration. The conclusion there is that, unless we know otherwise, we apparently do not imagine that changes in a person's knowledge as to whether Ingrid is Swedish can occur between the intervals.

When we put these two conclusions together with what we have said so far, we make a prediction. Consider the sentences in (82a) and (83a), which in some sense are parallel to the sentences we started out with in this section. On assumptions that can be reconstructed easily enough from the discussion thus far, one possible lf for (82a) - abbreviated in (82b) - yields the proposition in (82c), and similarly one possible If for (83a) - abbreviated in (83b) - yields the proposition in $(83 \mathrm{c})$. The If for $(82 \mathrm{a})$ says that, at each of the relevant time intervals, there is some secretary who knows at the time whether Ingrid is Swedish. The If for (83a) says that, at each of the relevant time intervals, there is some secretary who knows at the time whether Ingrid is on the phone (at the time).

(82) a. A secretary always knows whether Ingrid is Swedish.

b. always ${ }_{1}$ [VP [DP $a_{2}$ secretary $\left.e_{1}\right]$ [ $t_{2}$ knows whether In. is Sw. $\left.e_{1}\right]$ ]

c. $\lambda w$. For all intervals $t$ in the set of intervals that the context makes salient, there is some individual who, in w, is a secretary for the duration of $t$ and knows for the duration of $t$ whether Ingrid is Swedish

(83) a. A secretary always knows whether Ingrid is on the phone.

b. always 1 [vp [DP $a_{2}$ secretary $\left.e_{1}\right]$ [ $\mathrm{t}_{2}$ knows whether In. is on the ph. $\left.\mathrm{e}_{1}\right]$ ]

c. $\lambda w$. For all intervals $t$ in the set of intervals that the context makes salient, there is some individual who, in w, is a secretary for the duration of $t$

argued that another kind of If is available, and so the first course is open. In the case of sentences like Some secretary..., perhaps this option is not available.)

${ }^{14}$ Parallel sentences with quantifiers like half the time might be of use in verifying this claim. 
and knows for the duration of $\mathrm{t}$ whether Ingrid is on the phone

The prediction is this: in the absence of information about what time intervals are under consideration, it will not be natural to take (82a) to express the proposition that derives from that first lf; by contrast, it should be natural to take (83a) to express the parallel proposition that derives from the second If. In other words, the "semantic partition" difference that we find between A secretary is always Swedish and A secretary is always on the phone should be preserved across this kind of attitude context. (Why do we make this prediction? Because, if at one time interval we can find a secretary who knows whether Ingrid is Swedish, then we should also be able to find such a secretary at another time interval - the very same secretary. So the relevant If for $(82 \mathrm{a})$ will violate $(\mathrm{P})$. But if at one time interval we can find a secretary who knows whether Ingrid is on the phone, there is no guarantee that we can find such a secretary at another time interval. So the relevant If for (83a) will not violate (P).) This prediction seems to me to be correct. This is of interest because, contrary to what is often claimed, the ability to use Ifs like those in (82b)-(83b) apparently does not depend on the identity of the matrix verb: the matrix verb is the same (know) in both lfs, but it looks as though the first lf is usable while the second is not.

\section{Concluding remarks}

In this paper, I have argued for a conception of grammar under which principles of use may prevent us from availing ourselves of representations that we nonetheless have the resources to generate. I pointed out specifically that, since principles of use may sometimes block the use of lfs, we have to be careful when arguing that a sentence does not admit such and such an If.

I tried to motivate a particular principle of use, $(\mathrm{P})$, and my concern was to explore its effects. The questions that arise are the usual ones. Are there alternative lines of explanation that would have accounted for the same facts that I used (P) to account for? If indeed a theory that incorporates a principle like $(\mathrm{P})$ is on the right track, is the relevant principle really $(\mathrm{P})$, or is there a better way of formulating it? Does the principle follow from anything? Does it relate in any way to other principles of grammar that we know about?

To motivate (P), I used the fact that the sentence The student who finished first was always Swedish is unsuited to communicate the nationality of a certain student of whom it is known that she finished first each time. What other lines of explanation might one pursue to account for this fact? On the one hand, one might conjecture that the semantics of sentences like these is not what I claimed, and that the sentence's interpretation alone renders it incompatible with the situation in question. On the other hand, one might attribute the responsibility for this fact to other principles of use. One position to take, for example, is that it follows as a quantity implicature from the use of the sentence that the same student did not finish first each time. I can't address all the different options, but I am skeptical of this last position. To take this position is in part to say that we have a systematic way of generating alternatives to sentences like the one at issue, and that in the case of this sentence, the procedure will yield a sentence that is logically stronger and that entails that the same student did finish first every time. (One such alternative sentence might be: The student who always finished first was (always) Swedish.) I am skeptical because I do not see exactly how this procedure for generating alternatives would work.

Assuming something like (P) is on the right track, is the formulation of the principle in need of refinement? Probably. For one thing, some provision has to be made somewhere for the communicative intentions of the speaker: while in the scenario considered it is odd to utter 
The student who finished first was always Swedish in order to communicate the student's nationality, it is not so odd to utter the sentence in order to communicate a simple statistical generalization. Maybe a better formulation of the principle would reflect this. Apart from this, I think there is a further inadequacy with $(\mathrm{P})$ and $(\mathrm{Q})$ as they stand now. They predict that sentences of the kind in (84) should have the same status, when my intuition is that (84b) is less bizarre than (84a). (While I haven't come up with a context in which (84b) sounds perfect, my impression is that it evokes the kind of scenario in which someone checks every so often to see whether Napoleon has died.) They predict that the two sentences should have the same status because, in both cases, one can determine the sentence's truth by determining the truth of a domain-variant whose domain consists of a single time interval - in the case of (84a), the relevant time interval is the earliest one in the contextually salient set, and in the case of (84b) it is the last one. Accounting for the difference between (84a) and (84b) would certainly mean revising $(\mathrm{P})$, perhaps in such a way as to take into account the chronological order (or some other natural ordering) of the time intervals in the domain of quantification. ${ }^{15}$

a. Napoleon was always dead.

b. Napoleon was never dead.

There is another potential refinement worth mentioning: since my concern in this paper has been exclusively with adverbial quantifiers, I have formulated (P) to account only for facts involving adverbial quantifiers, but naturally $(\mathrm{P})$ should be extended to cover parallel facts involving quantifier phrases in other positions. If I tell you (85) alluding to the Marx Brothers, that sounds as odd as telling you The student who finished first was always Swedish when we know that the same student finished first each time. Presumably it sounds odd for the same reason.

\section{Each one's mother was named Minnie.}

Is there any connection between the principle of use that I have argued for, and anything else that we know about? As a pragmatic principle, $(\mathrm{P})$ looks very different in character from certain others that have been posited, in that it compares propositions that are equally informative. Perhaps it could be viewed as a subcase of Grice's Maxim of Manner ("Be brief.") (Its effect is radically different from the effect of the Maxim of Quantity, since, of the propositions it compares, it instructs the speaker to reject the logically stronger ones.) As for the facts that $(P)$ is designed to account for, there do seem to be facts that bear a surface similarity: the bizarreness of sentences like those in (86) is reminiscent of the bizarreness of the familiar sentences in (87).

a. ?? At that time, Ingrid was Swedish.

b \# On Tuesday, the student who finished first was Swedish. (given our second scenario)

\footnotetext{
${ }^{15}$ There are many candidates to think about. Here is an example (whose predictions I haven't thought about). Starting from a salient (strong) linear ordering of the intervals in the domain of quantification of [[ QUANT $_{j}$ $\alpha \mathrm{J}]^{\mathrm{g}}$, establish a corresponding ordering of those domain-variants whose domain consists exclusively of a single element of that set. (For instance, if the domain of (84a) is \{March 1810, April 1810, May 1810,... \}, order $\lambda w$. in $w$ Napoleon was dead for the duration of March 1810 before $\lambda w$. in $w$ Napoleon was dead for the duration of April 1810 before $\lambda w$. in $w$ Napoleon was dead for the duration of May 1810, etc.) New principle: Don't use $\left[\left[\text { QUANT }_{i} \alpha\right]\right]^{\mathrm{g}}$ if the truth of one of these domain-variants guarantees the truth of the next domain-variant in the order.
} 
a. ?? Ingrid was always Swedish.

b. \# The student who finished first was always Swedish.

(given our second scenario)

(P) does not account for the bizarreness of the sentences in (86). Since it is tempting to think that the problems with (87) can be reduced to the problems with (86), superficial similarities like these might lead one to pursue an approach very different from the one I have taken here. ${ }^{16,17}$

\section{References}

de Hoop, H./ H. de Swart (1989): Over Indefiniete Objecten en te Relatie tussen Syntaxis en Semantiek. Glot 12, 19-35.

Kratzer, A. (1995): Stage-Level and Individual-Level Predicates. In: G. Carlson and F.J. Pelletier (eds.) The Generic Book. Chicago: University of Chicago Press.

Musan, R. (1995): On the Temporal Interpretation of Noun Phrases. PhD diss., MIT.

Percus, O. (1997): Aspects of $A$. PhD diss., MIT.

Percus, O. (1998): A Somewhat More Definite Article. In D. Strolovitch and A. Lawson (eds.) Proceedings of SALT VIII. Ithaca NY: CLC Publications (Cornell).

Stalnaker, R. (1979): Assertion. In: P. Cole (ed.) Syntax and Semantics 9: Pragmatics, 315-332. New York: Academic Press.

de Swart, H. (1991): Adverbs of Quantification: A Generalized Quantifier Approach. PhD diss., Rijksuniversiteit Groningen.

\footnotetext{
${ }^{16}$ Some inspiration for an alternative approach might come from the idea that verifying sentences like those in (87) involves verifying many nonquantificational sentences of the kind in (86).

${ }^{17}$ It is natural to diagnose the oddness of (86) as resulting from a conflict with a quantity implicature. To evaluate this diagnosis, it might help to have a good description of the conditions of use of sentences with temporal adverbs. I think giving such a description is not so straightforward (Percus 1997 contains an attempt, and lists some of the relevant facts).
} 